\title{
The distributed particle detectors and data acquisition modules for Extensive Air Shower measurements at "HT-KZ" experiment
}

\author{
A. Duspayev ${ }^{1}$, A. Batyrkhanov, T. Beremkulov, D. Beznosko, A. lakovlev, M. \\ Yessenov \\ Nazarbayev University \\ 53 Kabanbai Batyr ave, Astana, KZ \\ E-mail: alisher.duspayev@nu.edu.kz
}

\section{R. U. Beisembaev, V. V. Zhukov}

P. N. Lebedev Physical Institute of the Russian Academy of Sciences

53 Leninskiy Prospekt, Moscow, Russia

\begin{abstract}
"HorizonT-Kazakhstan" (HT-KZ) is an extensive air shower new detector system of a new type to be constructed at Nazarbayev University (NU), Astana, KZ. It is based on the idea of a previous generation detector that is located at Tyan-Shan high-altitude Science Station of the Lebedev Physical Institute of the Russian Academy of Sciences at approximately 3340 meters above the sea level. It will consist of 8 independent modules distributed on the roofs of NU with 1-2 ns time resolution. The purpose is to register Extensive Air Showers (EAS) coming from a wide range of zenith angles. The measurements of the time characteristics of the EAS are to be taken simultaneously at up to eight registration points separated by the distance up to one kilometer. HT-KZ development is very important step in the EAS research area, especially, in the presence of the latest discoveries, such as multi-modal events This article presents the current system development state, the R\&D work of the system modules using the independent particle detection modules. The progress in development of the distributed DAQ system and the event synchronization system will be discussed as well.
\end{abstract}

International Conference on New Photo-detectors

PhotoDet 2015

6-9 July 2015

Moscow, Troitsk, Russia

${ }^{1}$ Speaker 


\section{Introduction}

In the field of High Energy Physics (HEP) today there are several open topics that are left. Out of them, one of the largest remaining mystery is the origin and the nature of the Ultra-high energy Cosmic Rays (UHECR).

The purpose of this project is to construct the HT-KZ detector system of the UHECRs with FIAN Tyan Shan high-altitude Science Station (FTSHASS) in order to study in detail the ground-breaking phenomena of the multi-modal events that possibly indicate the new particle discovery and to measure the directions to the origins of the UHECRs that are being currently studied.

The currently functioning system called HorizonT has both the optimal high-altitude location at the shower development maximum ( 3400 m above sea level in Tyan-Shan mountains, Kazakhstan), and <30ns time resolution that allows to study the details of the UHECR signal and to measure the angular coordinates of their origin.

This high time resolution, as compared to similar detector systems, has allowed for the preliminary discovery of the multi-modality within the UHECR. It can indicate that multiple UHECRs are arriving at the same time from the same source. Thus, HT-KZ is designed with higher time resolution then HorizonT in order to verify and further study this phenomenon in detail.

\subsection{Current HorizonT}

The FTSHASS was established in 1958 as a station devoted to studying of the cosmic rays in a wide energy range from the galactic and extra-galactic sources. Since then, the station personnel has accumulated a vast amount of knowledge and experience and has participated in a large number of different ground-breaking experiments over the course of the years. The study of time characteristics of the muon component was also carried our for many years [1-2].

The current HorizonT system consists of three Cerenkov light detectors (Figure 1, left) and seven working muon registration points (Figure 1, right) that are separated up to $500 \mathrm{~m}$ distance from the center point (points 8 to 10 are planned as future extensions).
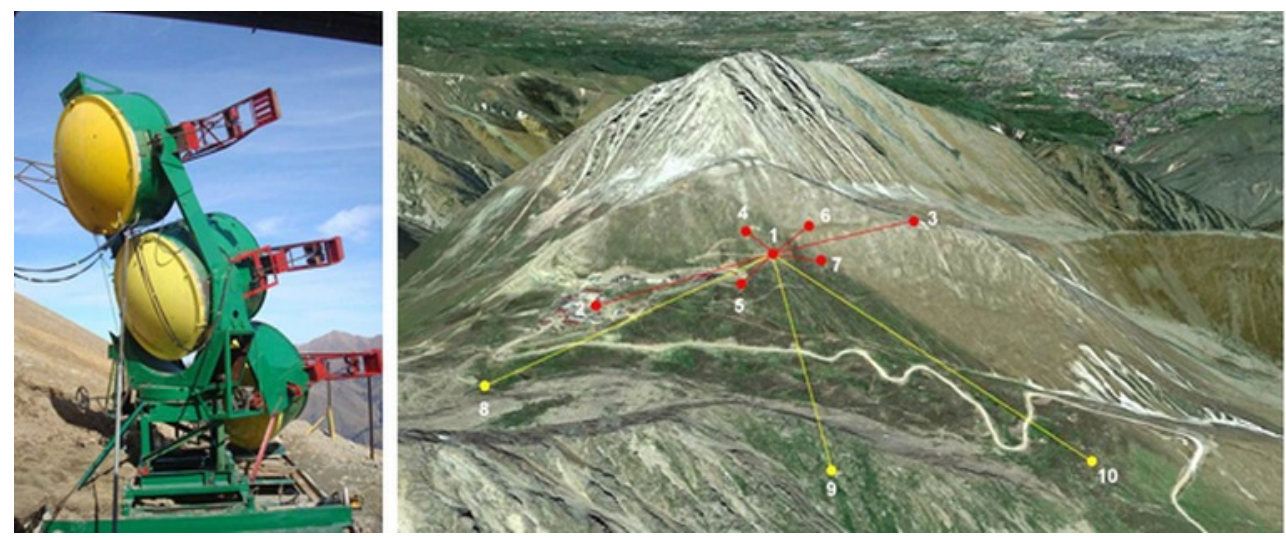

Figure 1: HorizonT prototype: Cerenkov light detectors (left) and muon registration points location (right) 
The methodology of the current HorizonT detector system is to look at the EAS from the UHECR near the horizon (up to $85^{\circ}$ from zenith) and to obtain the coordinates of the incoming UHECR using the timing resolution from the EAS disk reaching the detector, hence the name of the detector - HorizonT, time measurements at the (near) horizon level. In addition, the Cerenkov light detectors provide the detection of the Cerenkov light from the different EAS components (EM and muonic), a capability that will not be used in HT-KZ as it will be installed at the sea level.

\section{HT-KZ Detector System Design}

The HT-KZ detector system is envisioned to have a time resolution on the order of 1-2 ns using advanced electronics and data processing, and the area coverage by a grid of 8 detectors approximately every $150-200 \mathrm{~m}$ in a rectangular shape. The location for this detector is to be at NU. Detection points will be installed at the roof-tops of the university blocks (Figure 2) with the access to network and electric power. The location of the university at about sea level does not pose a problem for the detection of EAS from so high-energy parent particle of $>10^{17} \mathrm{eV}$.

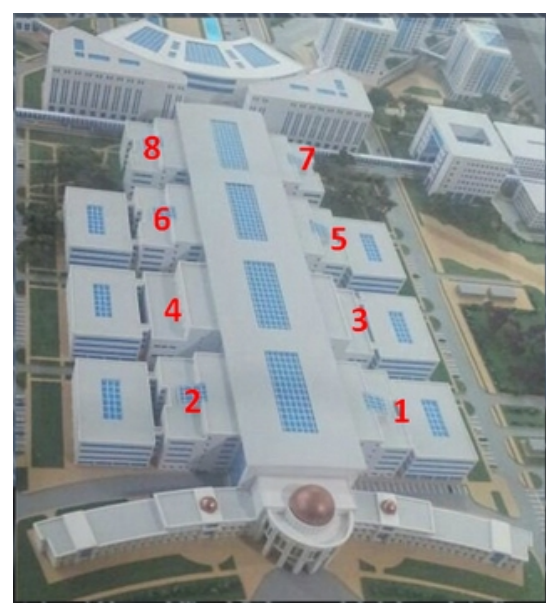

Figure 2: Schematic of the module location on the roof of $N U$.

\subsection{EAS Simulations}

Final properties of detector modules and their positions is a subject to further simulation studies (using CORSIKA simulation software package [3]).

Figure 3 shows the charged particle density distribution in EAS disk at the sea level for the $10^{17} \mathrm{eV}$ energy of the parent particle as a function of the distance from the disk center. As we can see, the particle density is dropping significantly after about $200 \mathrm{~m}$, thus the suggested detector separation of 150-200 $\mathrm{m}$ that is driven by building geometry is sufficient for the EAS detection at this energy. The simulation also sets limits to the size of the detectors to be discussed in the next subsection. It has been estimated that about 8000 events at energies above $\sim 10^{17} \mathrm{eV}$ will be detected. 


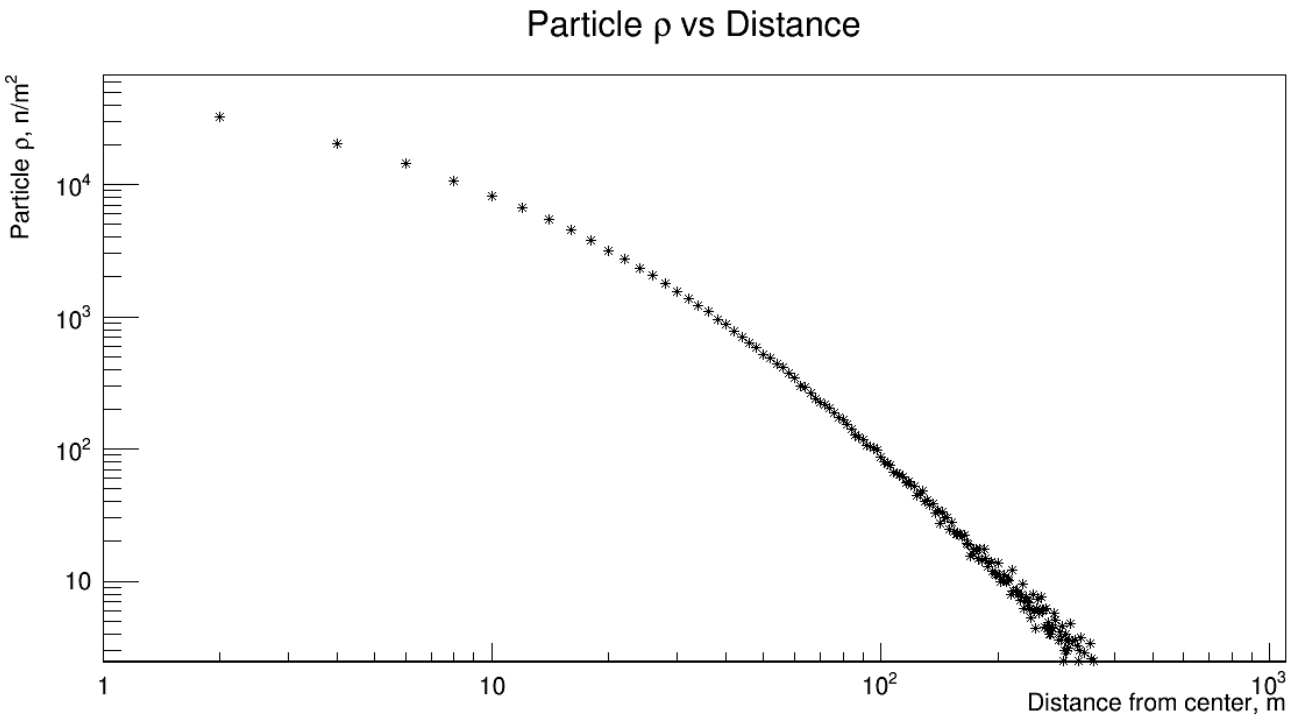

Figure 3: Charged particle density distribution in EAS disk at the sea level.

\subsection{Particle Detector Design}

Currently, a Hamamatsu [4] R7723 PMT is being identified as a most likely photo detector to be used. The requirements are the long term stability, temperature insensitivity, large dynamic range and fast response. The R7723 has a 1.7 ns rise time and $1.1 \mathrm{~ns}$ transit time spread; it it also relatively low-cost and, from our previous experience working with this PMT model, even the detectors from different production lines seem to be very closely matched in operational characteristics. As typical EAS signals could range from $\sim 10$ to several thousand near the center of the disk, a non-linearity of the response study for this PMT is currently planned.

To achieve the desired time resolution, the detection medium also has to be fast. The tests are in the progress using the aforementioned PMT and the following possibilities for the active material: $3 \mathrm{~cm}$ thick optical glass for Cerenkov light only, fast Eljen [5] EJ-230 scintillator (rise time $0.5 \mathrm{~ns}$, decay time $1.5 \mathrm{~ns}$ ) and Eljen EJ-299-15 Cerenkov radiator plastic with decay time of 1.9 ns. The EJ-200 (rise time 0.9 ns, decay time $2.1 \mathrm{ns)} \mathrm{that} \mathrm{is} \mathrm{considered} \mathrm{'standard'} \mathrm{for} \mathrm{Eljen}$ and similar to standards of other companies (e.g. BC408) in light yield and timing will be used as a reference. The dimensions of the medium are driven by both the simulation and by the light scattering time as to keep the time resolution, so the best value seems to lie between $30-50 \mathrm{~cm}$ for a rectangular shape scintillator.

\subsection{DAQ System}

To accommodate the distributed design and the time resolution requirements, a fast standalone CAEN [6] DTT 5743 ADC model was chosen. With up to $3.2 \mathrm{Gs} / \mathrm{s}$ acquisition resolution (planned to use $0.8 \mathrm{Gs} / \mathrm{s}$ ) and ability for self-triggering, this 8-channel ADC seems to fit well into the module design. Trigger level and logic are software controlled thus could be adjusted remotely; the resolution is 12 bit with the signal detection up to $2.5 \mathrm{Vpp}$. Dead time $125 \mathrm{~ms}$ that is $\sim 1 \%$ from the rate of the expected background events $(0.1 \mathrm{~Hz})$. The unit also has $40 \mathrm{bit}$ internal time stamp providing 5 ns resolution that is lower then envisioned thus additional 
external scheme will be used (still under R\&D). Another option of this ADC is that one or more channels can be used as a pulse generator, thus making easy integration of the particle detector calibration subsystem (e.g. LED) into the module.

\subsection{Detector Module Design}

A Module is a detector part meant for independent operation as a part of the whole system. It will consist of 3 particle detectors with their plains oriented along $\mathrm{x}, \mathrm{y}$ and $\mathrm{z}$ axes, ADC, computer, sync clock module and optional wireless router and solar panel (accumulator battery and power regulator as well) for installation in the locations without network and power feed. The design is to be waterproof and dust resistant for prolonged autonomous operations.

\section{Conclusion}

HT-KZ detector system is a work in progress. A particle detector with high time resolution is always a challenge and requires the use of the fast photo detector and a fast detection medium with as short interconnections between them as possible. In addition, DAQ system must be located on-site per each module and be able to synchronize with the ns precision. These and other challenges such as calibration, linearity and system stability are all part of the HT-KZ R\&D process currently in progress at Nazarbayev University, KZ.

\section{References}

[1] Beisembaev R. U., et al., Muons of extra high energy horizontal EAS in geomagnetic field and nucleonic astronomy, in proceedings of 24th ICRC (646-649)

[2] R.U. Beisembaev et al., EAS late muons and showers with two fronts, Nucl. Physics. 72, (11)

[3] D. Heck, J. Knapp, J.N. Capdevielle, G. Schatz, T. Thouw. CORSIKA: A Monte Carlo Code to Simulate Extensive Air Showers, Forschungszentrum Karlsruhe Report FZKA (6019)

[4] Hamamatsu Corporation. 314-5,Shimokanzo, Toyooka-village, Iwatagun,Shizuoka-ken, 438-0193 Japan.

[5] Eljen Technology. 1300 W. Broadway, Sweetwater, Texas 79556, United States

[6] CAEN S.p.A. Via della Vetraia, 11, 55049 Viareggio Lucca, Italy. 